\begin{abstract}
TITLE:
PERFORMANCE OF THE RAYLEIGH TASK BASED ON THE POSTERIOR PROBABILITY OF TOMOGRAPHIC RECONSTRUCTIONS

AUTHOR(S): Kenneth M. Hanson

Submitted to: IEEE Medical Imaging Conference

Santa $\mathrm{Fe}$, NM

November 8-9, 1991

\section{DISCLAIMER}

This report was prepared as an account of work sponsored by an agency of the United States Government. Neither the United States Government nor any agency thereof, nor any of their employees, makes any warranty, express or implied, or assumes any legal liability or responsibility for the accuracy, completeness, or usefulness of any information, apparatus, product, or process disclosed, or represents that its use would not infringe privately owned rights. Reference herein to any specific commercial product, process, or service by trade name, trademark, manufacturer, or otherwise does not necessarily constitute or imply its endorsement, recommendation, or favoring by the United States Government or any agency thereof. The views and opinions of authors expressed herein do not necessarily state or reflect those of the United States Government or any agency thereof.
\end{abstract}




\title{
Performance of the Rayleigh Task Based on the Posterior Probability of Tomographic Reconstructions
}

\author{
K.M. Hanson \\ Los Alamos National Laboratory \\ Los Alamos, NM 87545 \\ K.J. Myers \\ Center for Devices and Radiological Health, FDA \\ 12720 Twinbrook Parkway Rockville, MD 20857
}

\begin{abstract}
We seek the best possible performance of the Rayleigh task in which one must decide whether a perceived object is a pair of Gaussian-blurred points or a blurred line. Two Bayesian reconstruction algorithms are used, the first based on a Gaussian prior-probability distribution with a nonnegativity constraint and the second based on an entropic prior. In both cases, the reconstructions are found that maximize the posterior probability. We compare the performance of the Rayleigh task obtained with two decision variables, the logarithm of the posterior probability ratio and the change in the mean-squared deviation from the reconstruction. The method of evaluation is based on the results of a numerical testing procedure in which the stated discrimination task is carried out on reconstructions of a randomly generated sequence of images. The ability to perform the Rayleigh task is summarized in terms of a discrimination index that is derived from the area under the receiver-operating characteristic (ROC) curve. We find that the use of the posterior probability does not result in better performance of the Rayleigh task than the mean-squared deviation from the reconstruction.
\end{abstract}

\section{Introduction}

Appropriate measures of the quality of reconstructed images should ultimately be related to how well the images can be used to make decisions or estimate relevant physical quantities. The performance of imaging tasks is basically a statistical matter, not only when the data are degraded by random noise, but also when many randomly placed objects are reconstructed from limited, noiseless data. As such, a reconstruction technique cannot be judged on the basis of a reconstruction of only one scene. We use a method recently introduced to judge the quality of reconstruction by numerically evaluating task performance for a specified imaging situation [1.2]. The method consists of a Monte Carlo simulation of the entire imaging process including random scene generation, data taking, re. construction, and performance of the specified task. An

- This work was supported in part by the United States Department of Energy under contract number W-7405-ENG-36. essential aspect of this method is that the evaluation is based on many randomly generated scenes, leading to a statistically significant estimate of performance. Ve use the above technique to evaluate the performance of two reconstruction algorithms, both based on a Bayesian method in which the prior knowledge that the reconstruction mus $\hat{\imath}$ be nonnegative is employed. Such a constraint has been shown to be of great value in improving task performance when dealing with a paucity of data $[1,2]$ for as simple a reconstruction algorithm as the algebraic reconstruction technique (ART) [3]. Besides using the now-familiar entropy prior, we also reintroduce the classic Gaussian or normal prior probability distribution, but with the twist of an enforced nonnegativity constraint.

\section{The Bayesian Method}

The basis of the Bayesian approach is the posterior probability. which is assumed to summarize the complete state of knowledge after a set of measurements has been taken. The posterior probability properly combines prior information about the object being examined with the new information provided by the measurements.

\subsection{Posterior Probability}

We assume that the measurements, represented by a vector of length $M$, can be written as

$$
\mathbf{g}=\mathbf{H} \mathbf{f}+\mathbf{n},
$$

where $\mathrm{f}$ is the original image vector of length $\mathrm{X}, \mathrm{n}$ is the random noise vector, and $\mathbf{H}$ is the measurement matrix. Because the probability is a function of continuous parameters, namely the $N$ pixel values of the image and the $M$ data values, it is actually a probability density, designated by a small $p()$. By Bayes' theorem the negative logarithm of the posterior probability is given by

$$
\circ(\mathbf{f} . \mathbf{g}) x-\log [p(\mathbf{f} \mid \mathbf{g})]=-\log [p(\mathbf{g} \mid \mathbf{f})]-\log [p(\mathbf{f})] .
$$

where the first term is the probability of the observed data for any particular image f. called the likelihood, and the second term is the prior probability of the image f. For 
additive Gaussian noise, the negative $\log$ (likelihood) is just half of chi-squared

$$
-\log [p(\mathbf{g} \mid \mathbf{f})]=\frac{1}{2} \chi^{2}=\frac{1}{2}(\mathbf{g}-\mathbf{H} \mathbf{f})^{\mathrm{T}} \mathbf{R}_{\mathbf{n}}^{-1}(\mathbf{g}-\mathbf{H f}),
$$

where $\mathbf{R}_{\mathbf{n}}$ is the known noise covariance matrix. To simplify matters, we will make the siandard assumption that the measurement noise is stationary and uncorrelated, so $\mathbf{R}_{\mathbf{n}}=\operatorname{diag}\left(\sigma_{\mathbf{n}}^{2}\right)$, where $\sigma_{\mathbf{n}}$ is the rms deviation of the noise.

We use two relatively uninformative priors. The first is a Gaussian distribution, whose negative logarithm may be written as

$$
-\log [p(\mathbf{f})]=\frac{1}{2}(\mathbf{f}-\overline{\mathbf{f}})^{\mathrm{T}} \mathbf{R}_{\mathbf{f}}^{-1}(\mathbf{f}-\overline{\mathbf{f}}),
$$

where $\bar{f}$ is the mean and $R_{f}$ is the covariance matrix of the prior-probability distribution. As we have done before [1] we invoke the prior knowledge that the image $f$ cannot possess any negative components and institute nonnegativity as a separate constraint. We simplify this expression by setting $\mathbf{R}_{\mathbf{f}}=\operatorname{diag}\left(\sigma_{\mathbf{f}}^{2}\right)$, where $\sigma_{\mathbf{f}}$ is the rms deviation of the prior-probability distribution.

For the second prior, we use one based on entropy [5];

$$
-\log [p(\mathbf{f})]=-\alpha \sum_{i}\left[f_{i}-\tilde{f}_{i}-f_{i} \ln \left(f_{i} / \tilde{f}_{i}\right)\right] .
$$

The sum is over all pixels in the image and $\tilde{f}_{i}$ is aptly called the default value for the $i$ th pixel representing the prior model for the reconstruction. The maximum-entropy prior implies a reconstruction that is nonnegative.

\subsection{Reconstruction Problem}

In the reconstruction problem, we seek to estimate all pixel values in the original scene. An appropriate Bayesian solution to this problem is the image that maximizes the posterior probability or, equivalently, minimizes the negative logarithm of the posterior probability. For the unconstrained MAP solution $\hat{\mathbf{f}}$, it is necessary that

$$
\nabla_{\mathbf{f}} \phi=\mathbf{R}_{\mathbf{f}}^{-1}(\mathbf{f}-\overline{\mathbf{f}})+\mathbf{H}^{\mathrm{T}} \mathbf{R}_{n}^{-1}(\mathbf{g}-\mathbf{H} \mathbf{f})=0 .
$$

However, under the constraint that the solution should be nonnegative, the derivative with respect to $f_{i}$ must be zero only when $f_{i}>0$; a negative derivative is permissible on the boundary $f_{i}=0$. In computed tomography $(C T)$, the matrix operation $\mathbf{H}^{\mathrm{T}}$ is the familiar backprojection process. We employ the iterative method described by Butler, Reeds, and Dawson [4] to fiald the constrained MAP solution for the Gaussian prio:. See [6,7] for more details. For the entropic prior, we employ the proprietary code MEMSYS ${ }^{1}$. The choice of the strength of the prior has long been a topic of debate. The MEMSYS3 code purports to solve this problem through an approach called 'classic MaxEnt' [5], with its intrinsic determination of $\alpha$.

A consequence of the prior is to pull the reconstruction away from the actual value in the original image, an effect studied by Hanson [8] in unconstrained tomographic

\footnotetext{
${ }^{1}$ Maximum Entropy Data Consultants Ltd., Royston, England
}

reconstructions. The extent of this biasing effect depends on the relative weights of the two terms in Eq. (2). As the prior contribution vanishes, the MAP result approaches the maximum likelihood (or least-scquare residual) solution.

\subsection{Decisions about a Local Region}

Instead of asking for an estimate of the original image. suppose that we ask a different question: which of two possible objects exists in a specific region in the image? We denote the subimage contained within the local region of interest $\mathcal{D}$ by $\mathbf{f}_{\mathcal{D}}$ and the image exterior to $\mathcal{D}$ by $\mathbf{f}_{\mathcal{E}}$. Then we are asking whether the image is $f_{\mathcal{D}_{1}}$ or $\mathbf{f}_{\mathcal{D}_{2}}$ inside $\mathcal{D}$. Following Bayes, the decision should be made on the basis of the ratio of the posterior probabilities for these two possibilities. For the present purpose, we use for the decision variable

$$
\iota^{\prime} 21=\log \left[\frac{p\left(\hat{\mathbf{f}}_{\mathcal{E}}, \mathbf{f}_{\mathcal{D} 2} \mid \mathbf{g}\right)}{p\left(\hat{\mathbf{f}}_{\mathcal{E}}, \mathbf{f}_{\mathcal{D} 1} \mid \mathbf{g}\right)}\right] .
$$

by which is meant that the part of the image outside the analyzed region is fixed at the reconstruction value $\hat{f}$. With this approach we are ignoring some details in the Bayesian theory $[7,10]$. We designate this decision method as PP. To evaluate the posterior probability for the entropic prior, it is convenient to use the Gaussian approximation

$$
\phi(\mathbf{f}) \approx \phi(\hat{\mathbf{f}})+\frac{\alpha}{2} \sum_{i} \frac{\left(f_{i}-\hat{f}_{i}\right)^{2}}{\hat{f}_{i}}+\frac{1}{2 \sigma_{\mathrm{n}}^{2}}|\mathbf{H}(\mathbf{f}-\hat{\mathbf{f}})|^{2},
$$

which is valid near the maximum posterior-probability solution $\hat{\mathbf{f}}$.

An alternative decision variable is the change in the mean-squared deviation from the reconstruction, that is

$$
\psi_{21}=\left|\mathbf{f}_{1}-\hat{\mathbf{f}}\right|^{2}-\left|\mathbf{f}_{2}-\hat{\mathbf{f}}\right|^{2},
$$

which method we designate as MSDEV. This decision variable would be ideal if the fluctuations in the reconstruction were uncorrelated and stationary, which we know is not true.

\section{Rayleigh Task Performance}

A natural imaging task, named after Lo:d Rayleigh, is to determine whether an observed astronomical object is actually a pair of stars or a single star. Precisely stated, we will consider the task of distinguishing between a pair of point-like objects and a line, both convolved with a 2D Gaussian spread function. By specifying binary pairs that are farther apart than the width of the Gaussian and by specifying the alternative line to be similar in appearance, the decision must be made on the basis of more than an observed slight asymmetry; an honest dip must be observed between the two lobes of a reconstructed binary for it to be distinguished from a line. 


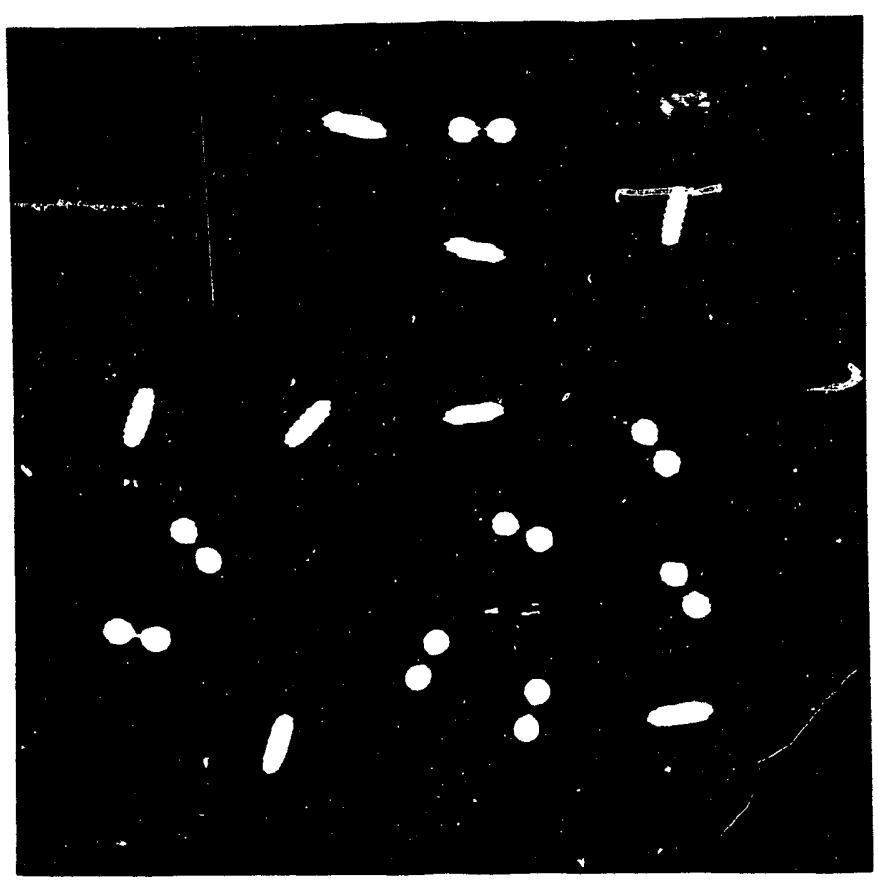

Figure 1: The first in a series of ten scenes used to test performance of the Rayleigh task. After projection meas'irements of these scenes are reconstructed. the decision maker must identify each object as either a binary pair or a bar.

\subsection{Monte Carlo Testing Procedure}

A Monte Carlo technique, one that employs pseudorandom numbers to generate its results. is used to simulate the entire imaging process from scene generation to the final task performance. The general strategy for simulation of the scenes, data taking, and reconstruction is straightforward [1,2]. The scenes used to test task performance are generated each with eight binary pairs and eight lines randomly placed and randomly oriented with the circle of reconstruction inscribed within a $128 \times 128$-pixel image. The binary pairs of points are separated by 6 pixels and the lines are 10.4 pixels long. Each of these types of objects is convolved with a symmetric 2D Gaussian function with a FWH.M of 4 pixels. The line length and amplitude are chosen to minimize the mean-square difference between the two possible objects. The purpose is to make the two objects similar in appearance so the decision must be made on the basis of the details of the image. not gross features such as integrated intensity [9]. The first of the series of images generated for testing performance of the Rayleigh task is shown in Figure 1. Ten such scenes are used in the present tests.

The Rayleigh task is carried out under two separate assumptions about the object. The first situation tested is when the two possible signals are assumed to be perfectly known, which we call the signal-known-exactly (SKE) case. Under the alternative assumption that the amplitude and asymmetry of the objects, as well as the background. are unknown beforehand, these parameters must be deter-

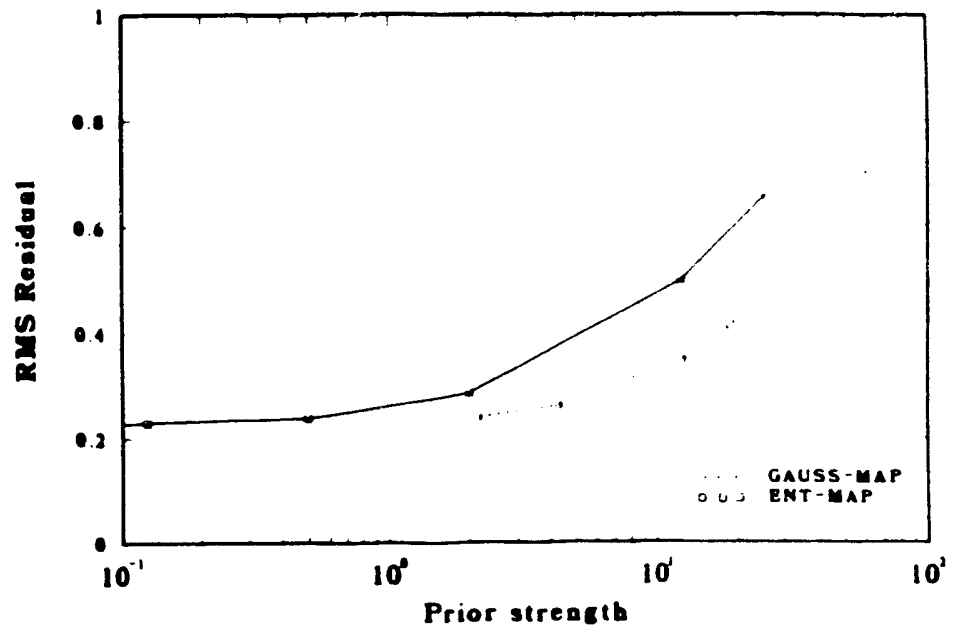

Figure 2: The rms residuals in the MAP reconstructions vs the strength of the prior for the two priors studied. Gaussian and entropic.

mined from the reconstruction. These three parameters are fit to minimize 0 . We call this situation the FIT case. For both of these situations, the region of analysis is a 16 pixel-diam circle, centered on the known position of the object. Two different decision variables are employed, as explained in Sect. 2.3.

Frequency histograms of the decision variable for each of the two possible classes of objects are formed. A receiver operating characteristic (ROC) curve is generated from these as described previously [1.2]. The area under the ROC curve is an appropriate measure of how well the distinction between the two hypotheses is made. A useful way to summarize the significance of the area is to quote the discrimination index $d_{A}$. An alternative index $d^{\prime}$ is based on the rms widths and separation of the two histograms. It is equal to $d_{A}$ when the histograms are Gaussian shaped. However, $d^{\prime}$ is not used here because the histograms of the decision variable may not be Gaussian. The index $d_{A}$ is preferable as it is invariant under any monotonic transformation of the decision variable. whereas $d^{\prime}$ is not.

\section{$4 \quad$ Results}

The main interest in this study is the behavior of $d_{t}$ as a function of the strength of the prior. Figure 2 shows the dependence of the rms residuals for the MAP reconstructions on the strength of the prior. which is taken to be $\left[n \sigma_{\mathrm{f}}^{-2}\right]^{-1}$ for the Gaussian prior and $\alpha /(n \bar{f})$ for the entropic prior, where $n$ is the number of views $(=8)$.

The results for task performance under the SKE assumption are plotted against the rms residuals in Figs. 3 and 4 . It is seen that decisions made on the basis of the posterior probability, $E_{q}$. ( $T$ ). are generally slightly less reliable than those made on the basis of the simpler mean-squared deviation, Eq. (9). Although not a large effect, this re- 


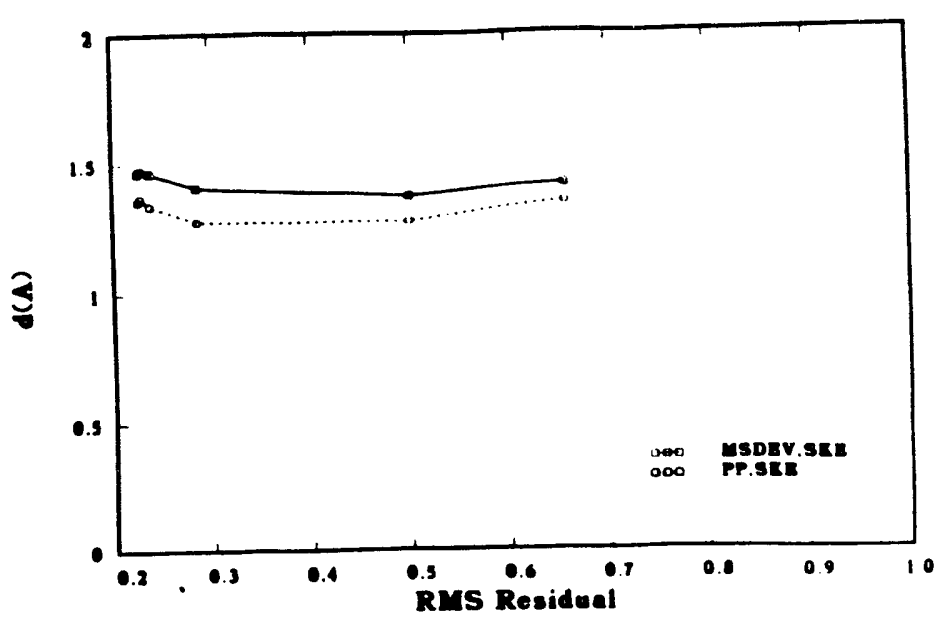

Figure 3: Discrimination index $d_{A}$ vs rms residual for the GAUSS-MAP algorithm when the signal is known exactly (SKE).

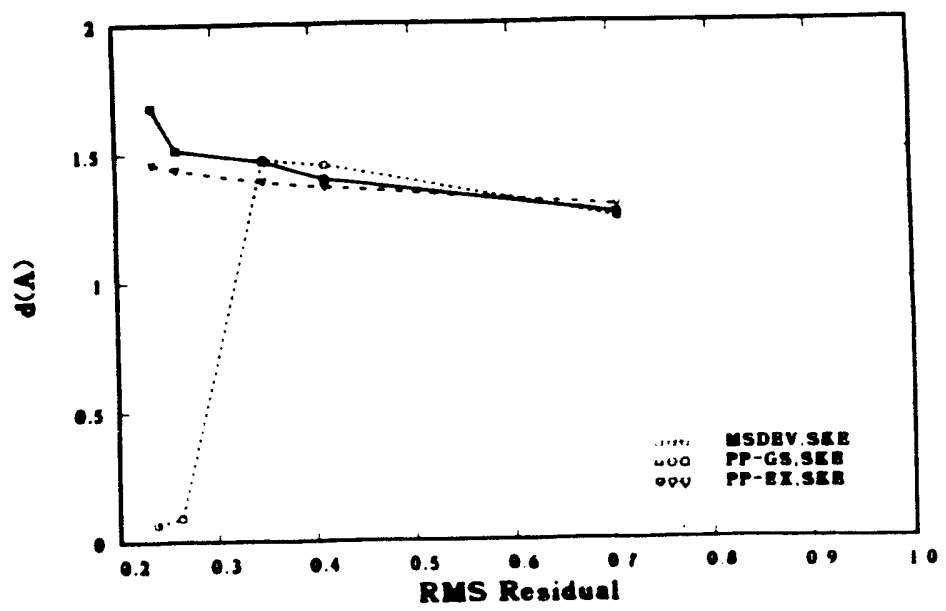

Figure 4: Discrimination index $d_{A}$ vs rms residual for the ENTROPY-MAP algorithm when the signal is known exactly (SKE).

sult is a bit surprising, because it seems to run contrary to the Bayesian formulation on which the reconstructions are based. Further investigation of this effect is needed. We observe a catastrophic failure of the Gaussian approximation to the entropic prior, Eq. (8), for small rms residuals. The use of the exact entropy expression, Eq. (5), labelled PP-EX,SKE in Fig. 4, overcomes this difficulty.

When the signal is not known exactly beforehand, but when three parameters need to be determined from the reconstructions, the $d_{A}$ index is generally diminished, as seen in Figs. 5 and 6 . This reduction is understandable, because less information is provided about the object. In this case, the posterior probability decision variable demonstrates a slight advantage over the mean-squared deviation for larger rms residuals. The GAUSS-MAP algorithm does not perform as well as the ENTROPY-MAP one for this more

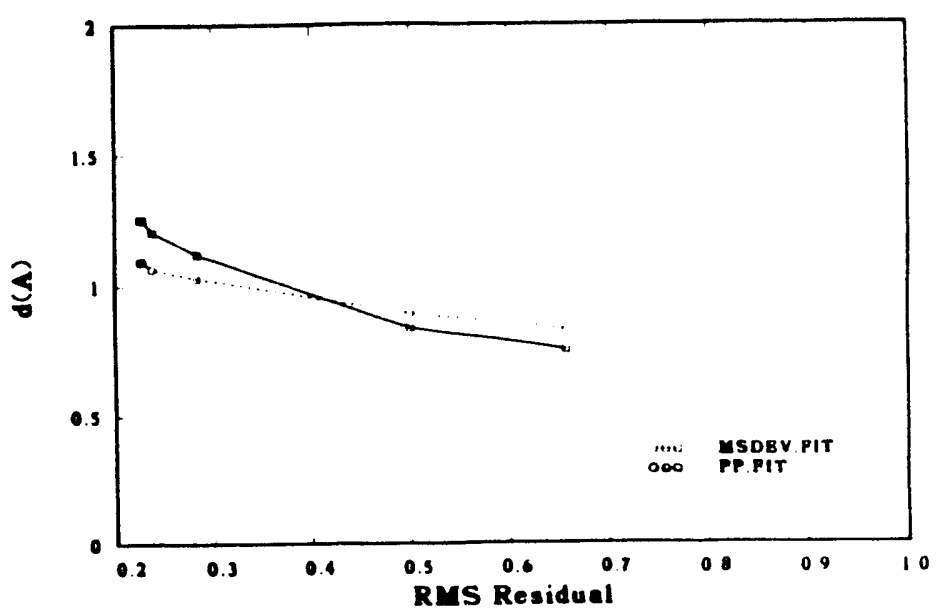

Figure 5: Discrimination index $d_{A}$ vs rms residual for the GAUSS-MAP algorithm when the amplitude, asymmetry, and background are fit.

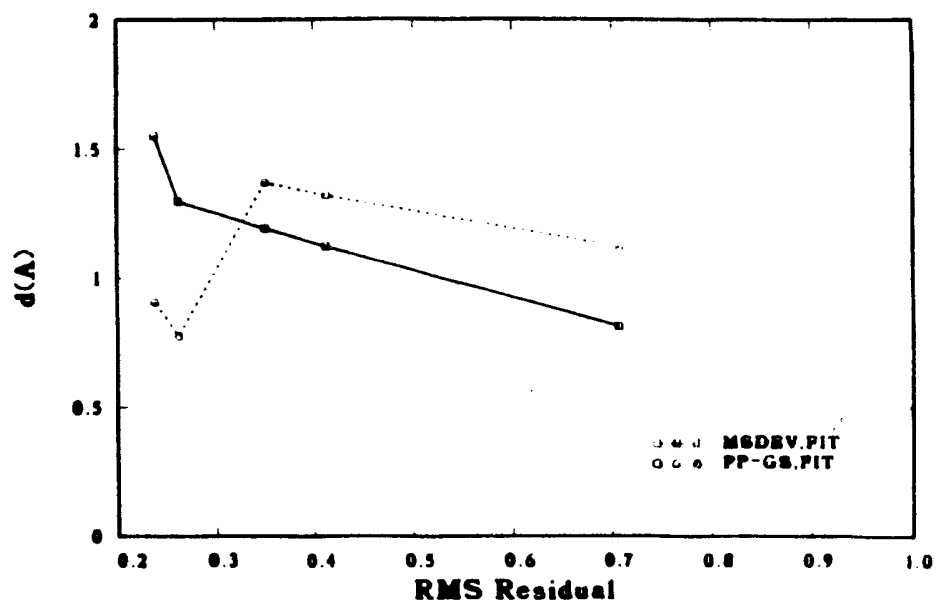

Figure 6: Discrimination index $d_{A}$ vs rms residual for the ENTROPY-MAP algorithm when the amplitude, asymmetry, and background are fit.

elaborate task. We note that the classic MaxEnt mode of MEMSYS3 selects an $\alpha$ value of 0.60 for these tests corresponding to an $\mathrm{rms}$ residual of 0.26 , which is the second leftmost point on the ENTROPY-MAP plots. It does not yield the best task performance. Rather, the best performance of the Rayleigh task is observed to occur at the smallest rms residual, which is closest to the maximumlikelihood limit.

\section{Acknowledgments}

The authors wish to acknowledge many provocative conversations with Robert $F$. Wagner. We have also benefitted greatly from conversations with Stephen F. Gull, John Skilling, and Charles E. Metz. This work was supported in part by the U.S. Department of Energy under contract 
number W-7405-ENG-36.

\section{References}

[1] K. M. Hanson: 'Method to evaluate image-recovery algorithms based on task performance'. Proc. SPIE 914, 336-343, 1988.

[2] I. M. Hanson: 'Method to evaluate image-recovery algorithms based on task performance: J. Opt. Soc. 7 A, 1294-1304, 1990.

[3] 'Algebraic reconstruction techniques for threedimensional electron microscopy and x-ray photography", J. Theor. Biol. 29, 471-481, 1970.

[4] J. P. Butler, J. A. Reeds, and S. V. Dawson: 'Estimating solutions for first kind integral equations with nonnegative constraints and optimal smoothing:. SIAM J. Numer. Anal. 18, 381-397, 1981.

[5] S. F. Gull: 'Bayesian inductive inference and maximum entropy', in Maximum Entropy and Bayesian Methods in Science and Engineering (Vol. 1), G. J. Erickson and C. R. Smith (ed.), Kluwer, Dordrecht, 53-74, 1989.

[6] K. M. Hanson and K. J. Myers: 'Rayleigh task performance as a method to evaluate image reconstruction algorithms', in Maximum Entropy and Bayesian Methods, IV. T. Grandy and L. H. Schick (ed.), Kluwer Academic, Dordrecht, 303-312, 1991.

[i] K. M. Hanson: 'Simultaneous object estimation and image reconstruction in a Bayesian setting', Proc. SPIE 1452, 180-191. 1991.

[8] K. M.Hanson: 'Object detection and amplitude estimation based on maximum a posteriori reconstructions', Proc. SPIE 1231, 164-175. 1990.

[9] K. M. Hanson: 'Variations in task and the ideal observer', Proc. SPIE 419, 60-67,1983.

[10] K. M. Hanson: Making binary decisions based on the posterior probability distribution associated with tomographic reconstructions', to be published in Maximum Entropy and Bayesian Methods in Statistical Analysis, G. J. Erickson and P. Neudorfer (ed.), Kluwer, Dordrecht, 1991. 

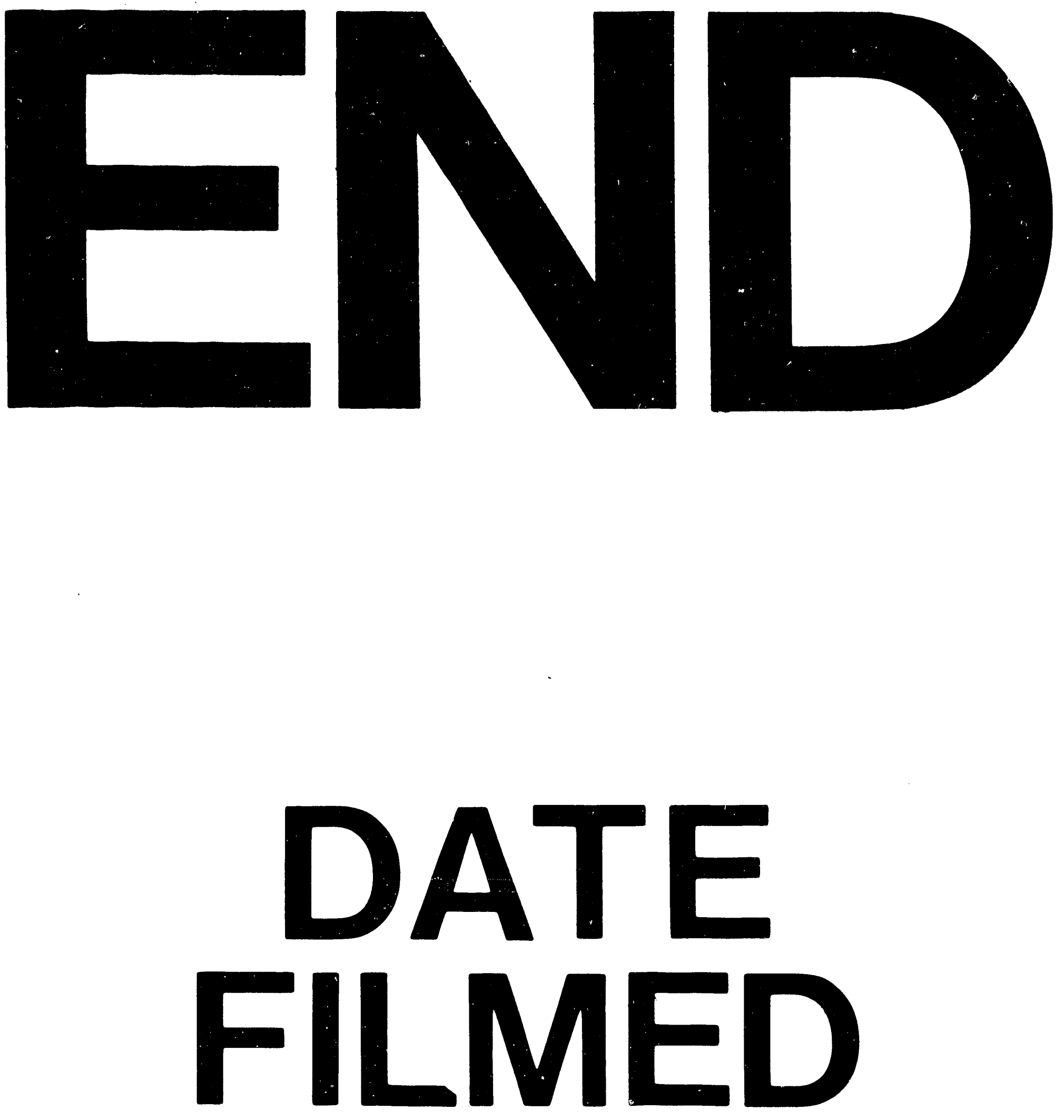

1

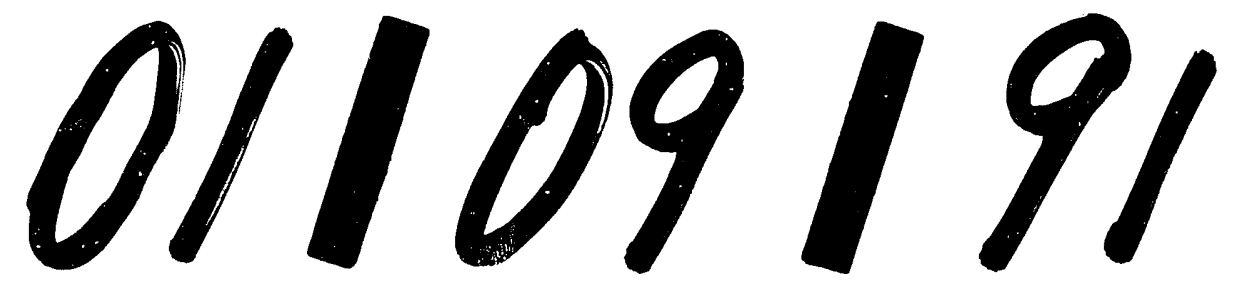

$\because$ 
